\title{
HDAC Activity Is Required for Efficient Core Promoter Function at the Mouse Mammary Tumor Virus Promoter
}

\author{
Sang C. Lee, ${ }^{1}$ Angeliki Magklara, ${ }^{2}$ and Catharine L. Smith ${ }^{1}$ \\ ${ }^{1}$ Department of Pharmacology and Toxicology, College of Pharmacy, University of Arizona, 1703 E. Mabel Street, \\ Tucson, AZ 85721-0207, USA \\ ${ }^{2}$ Department of Anatomy, UCSF Mission Bay, 1550 4th Street, San Francisco, CA 94158-2324, USA
}

Correspondence should be addressed to Catharine L. Smith, csmith@pharmacy.arizona.edu

Received 15 July 2010; Accepted 1 November 2010

Academic Editor: Minoru Yoshida

Copyright (c) 2011 Sang C. Lee et al. This is an open access article distributed under the Creative Commons Attribution License, which permits unrestricted use, distribution, and reproduction in any medium, provided the original work is properly cited.

\begin{abstract}
Histone deacetylases (HDACs) have been shown to be required for basal or inducible transcription at a variety of genes by poorly understood mechanisms. We demonstrated previously that HDAC inhibition rapidly repressed transcription from the mouse mammary tumor virus (MMTV) promoter by a mechanism that does not require the binding of upstream transcription factors. In the current study, we find that HDACs work through the core promoter sequences of MMTV as well as those of several cellular genes to facilitate transcriptional initiation through deacetylation of nonhistone proteins.
\end{abstract}

\section{Introduction}

Histone deacetylases (HDACs) comprise a large and varied family of proteins which are divided into four classes based on structural homologies [1]. Interest in their role in development, physiology, and cell biology has been intensified by the development of small molecule inhibitors of class I (HDACs 1-3, 8) and class II (HDACs 4-7, 10) family members as anticancer drugs. Two such inhibitors, Vorinostat and Romidepsin, are now approved for treatment of advanced cutaneous T-cell lymphoma and many more are being evaluated in clinical trials [2]. These drugs are also being considered for use in other diseases including cardiovascular, neurodegenerative, and inflammatory disorders [3-5]. In spite of their current and potential clinical use, the precise roles of HDACs in regulating cellular processes through protein acetylation are largely unknown. HDACs are thought to control cell growth and survival through both transcriptional and nontranscriptional pathways that may be partially specific to cell type (reviewed in [6]). Currently our lack of knowledge about the general and cell-specific functions of HDACs limits our ability to predict whether particular tumors will respond to HDAC inhibitors and which other therapeutics might work synergistically with these drugs in treating various cancers.

Long-standing models of the role of acetylation in transcription generally cast histone acetyltransferases (HATs) as coactivators and HDACs as corepressors [7]. This is based on the positive relationship between histone acetylation and transcription and the fact that many transcriptional activators recruit HATs to target promoters. In contrast, there is a negative correlation between histone hypoacetylation and transcription, and HDACs are often found in complexes that work to repress transcription. This model, however, is inadequate to explain all the roles of HDACs in transcription. Expression profiling of cells treated with HDAC inhibitors [8-14] shows that genes are both activated and repressed by these drugs. Gene repression is observed even within $2 \mathrm{~h}$ of treatment when effects on transcription are likely to be the direct result of HDAC inhibition rather than secondary effects dependent on prior changes in gene expression. In addition, studies of individual genes have demonstrated direct repressive transcriptional effects of HDAC inhibitors or knockdown of particular HDACs (reviewed in $[15,16]$ ). Furthermore, transcriptional activators such as glucocorticoid receptor (GR) and STATs 1, 2, and 5 have been shown 
to interact with HDACs at target genes to facilitate transcription [17-20]. Finally, a recent genomewide mapping study showed that in addition to HATs, HDACs are enriched at the promoters of active genes and are recruited along with HATs to transcriptionally inducible genes [21]. Taken together, this accumulated evidence indicates that HDACs serve an important role in the activation of transcription and/or maintenance of the activated state. However, the mechanisms by which HDACs facilitate transcription are poorly understood.

One example of a promoter that is repressed by HDAC inhibitors [22-26] or HDAC knockdown [17] is that of the mouse mammary tumor virus (MMTV), a well-studied model system for glucocorticoid receptor- (GR-) mediated activation of transcription. Initially, it was reported that transcriptional activation of this promoter by glucocorticoids was significantly impaired in the presence of HDAC inhibitors [23]. Later, we and others showed that basal MMTV transcription was also repressed $[22,24]$. We demonstrated that this repression was very rapid and potent but occurred independently of changes in histone acetylation, chromatin remodeling, or chromatin structure [22]. Our analysis of the sequences required for repression showed that sequences upstream of the TATA box were dispensable. We concluded that HDACs facilitated MMTV transcription through nonhistone proteins which are essential for efficient basal transcription. More recently, Qiu et al. [17] showed that HDAC1 can be found in GR complexes and is associated with the MMTV promoter in the presence and absence of glucocorticoids. In addition, they showed that siRNAmediated knockdown of HDAC1 impaired the ability of GR to activate the promoter. Together these data make a strong case that HDAC activity is required to facilitate both basal and activated transcription at the MMTV promoter.

In the current study, we have investigated the mechanism by which HDAC inhibition impairs basal transcription. Key to our findings was the development of an in vitro transcription system that recapitulates repression of MMTV transcription due to inhibition of HDAC activity. Surprisingly we find that the repression is conferred through the MMTV core promoter and involves impaired transcriptional initiation. Our results have revealed a novel role of HDAC activity in promoting transcription.

\section{Materials and Methods}

2.1. Cell Culture. Cell line 1470.2 is derived from C127i mouse mammary adenocarcinoma cells and contains stably replicating copies of a transcription unit consisting of the MMTV long terminal repeat (LTR) driving expression of the chloramphenicol acetyltransferase (CAT) gene. Both 1470.2 and Hela cells were maintained in Dulbecco's Media Essential Medium (DMEM), containing 10\% fetal bovine serum.

2.2. Plasmids. pMluc and $\mathrm{pMTV}(\mathrm{TATA} /+100)$ luc have been previously described [22]. pMTV(TATA-DPE)luc contains MMTV LTR sequences from -40 to $+32 \mathrm{bp}$. pMTV(TATAInr)luc contains MMTV LTR sequences from -40 to $+4 \mathrm{bp}$.
Both are cloned into pXP1, a promoterless luciferase reporter construct [27]. The luciferase constructs containing core promoters from cytomegalovirus (CMV), c-fos, interleukin 4 (IL4), and carbonic anhydrase 2 (CA2) were generated by cloning annealed oligonucleotides with sequences from the TATA box to Inr element for each gene into pXP1. pAdML was a gift from Dr. Gordon Hager (NCI, NIH, Bethesda, MD). It contains a $190 \mathrm{bp} \mathrm{G-free} \mathrm{cassette} 3^{\prime}$ to the Adenovirus Major Late (AdML) promoter. pMTV-InrAG, the MMTV template used for in vitro transcription, was constructed by subcloning annealed oligonucleotides containing MMTV LTR sequences ( -39 to $+5 \mathrm{bp}$ ) just $5^{\prime}$ of the 400 bp G-free cassette of $\mathrm{pC}_{2} \mathrm{AT} 19$ (a gift from Dr. Robert Roeder, Rockefeller University). The transcription start site of the MMTV promoter is a $\mathrm{G}$ nucleotide. It was modified to an A in pMTV-InrA-G for use with the G-free cassette. This modification did not affect core promoter activity under any of the conditions tested in this study (data not shown).

\subsection{Transfection Assays. DNA was transiently transfected into HeLa cells using the calcium phosphate method accord- ing to the manufacturer's protocol (Invitrogen, Carlsbad, CA). Two days after transfection, cells were treated and harvested as described previously [22]. Cell line 1470.2 was transfected by electroporation as described [22]. Treatment and harvest was carried out 1 day after transfection. Lysates from both cell lines were assayed for protein concentration and luciferase activity. Luciferase activities were normalized to protein concentration for each sample.}

2.4. Chromatin Immunoprecipitation (ChIP) Assay. The ChIP assay was performed as described by Magklara and Smith [28] with slight modification. Briefly, 1470.2 cells were grown to $95 \%$ confluency on $150 \mathrm{~mm}$ plates and treated with $50 \mathrm{ng} / \mathrm{ml}$ TSA or $100 \mathrm{nM}$ dexamethasone (Dex) for 15,30 , or 60 minutes. The cells were fixed with $1 \%$ formaldehyde for 10 minutes at $37^{\circ} \mathrm{C}$ and neutralized with $0.125 \mathrm{M}$ glycine. After collection of cells by scraping, they were washed $3 \mathrm{X}$ with cold PBS and incubated with lysis buffer (1\% SDS, $10 \mathrm{mM}$ EDTA, $50 \mathrm{mM}$ Tris $\mathrm{HCl}, \mathrm{pH} 8.0$ ) for 10 minutes on ice. After centrifugation cells were resuspended in $1.5 \mathrm{ml}$ lysis buffer and sonicated. Chromatin was precleared with a mixture of protein $A$ and $G$ agarose beads containing salmon sperm DNA. The supernatants were then incubated with 3 to $5 \mu \mathrm{g}$ 8WG16 RNA polymerase II antibody (Covance) for $16 \mathrm{~h}$ at $4^{\circ} \mathrm{C}$. Protein $\mathrm{A}$ and $\mathrm{G}$ agarose beads were added and incubation continued for $2 \mathrm{~h}$. Subsequently, chromatin-bead complexes were washed once each with low salt buffer $(0.1 \%$ SDS, $1 \%$ Triton-X100, 2 mM EDTA, 20 mM Tri-HCl, pH 8.0, $50 \mathrm{mM} \mathrm{NaCl})$, high salt buffer (0.1\% SDS, $1 \%$ Triton-X100, $2 \mathrm{mM}$ EDTA, $20 \mathrm{mM}$ Tri-HCl, pH 8.0, $500 \mathrm{mM} \mathrm{NaCl}$ ), and lithium chloride buffer $(250 \mathrm{mM} \mathrm{LiCl}, 1 \% \mathrm{NP} 40,1 \%$ deoxycholate, $1 \mathrm{mM}$ EDTA, $10 \mathrm{mM}$ Tris- $\mathrm{HCl}$ ) and twice with TE buffer. All buffers contained protease inhibitors. Bound chromatin was eluted successively with $1.5 \%$ SDS buffer $\left(1.5 \%\right.$ SDS, $\left.0.1 \mathrm{M} \mathrm{NaHCO}_{3}\right)$ and $0.5 \%$ SDS buffer $\left(0.5 \%\right.$ SDS, $\left.0.1 \mathrm{M} \mathrm{NaHCO}_{3}\right)$. Crosslinks were reversed by incubation at $65^{\circ} \mathrm{C}$ for $5 \mathrm{~h}$ in the presence of $200 \mathrm{mM} \mathrm{NaCl}$ 
and $10 \mu \mathrm{g}$ RNase A. Protein was digested at $45^{\circ} \mathrm{C}$ for $2 \mathrm{~h}$ in the presence of $10 \mathrm{mM}$ EDTA, $40 \mathrm{mM}$ Tris- $\mathrm{HCl}$ pH6.5, and $20 \mu \mathrm{g}$ proteinase K. DNA was extracted twice with phenolchloroform-isoamyl alcohol and precipitated with ethanol in the presence of glycogen. Detection of MMTV sequences was carried out by PCR analysis using the following primers: MMTV promoter $/ 5^{\prime}$ transcribed region-Forward5'TTTCCATACCAAGGAGGGGACAGTG 3' ${ }^{\prime}$, Reverse- $5^{\prime}$ CTTACTTAAGCCTTGGGAACCGCAA $3^{\prime}$, CAT coding region (CDS)-Forward-5' CCGTTTTCACCATGGGCAAA 3', Reverse-5' AAGCATTCTGCCGACATGGA $3^{\prime}$. In each experiment, cycle number was empirically adjusted so that amplification of the desired sequences was in the linear range. Generally, cycle number varied between 22 and 28 cycles.

2.5. HeLa Nuclear Extract Preparation. Hela S3 cell pellets were purchased from the National Cell Culture Center. Cultures of Hela S3 cells (5 liters at $0.5 \times 10^{6} \mathrm{cell} / \mathrm{ml}$ ) were treated with or without TSA $(50 \mathrm{ng} / \mathrm{ml})$ for $2 \mathrm{~h}$. Cells were pelleted and flash frozen prior to overnight shipping. Nuclear extracts were prepared by the method of Kusk et al. [29] with modification. All procedures were conducted at $4^{\circ} \mathrm{C}$ and all buffers used in the extraction procedure contained $500 \mu \mathrm{M}$ AEBSF, $150 \mathrm{nM}$ aprotinin, $1 \mu \mathrm{M}$ E-64, $0.5 \mathrm{mM}$ EDTA, and $1 \mu \mathrm{M}$ leupeptin. TSA $(50 \mathrm{ng} / \mathrm{ml})$ was included in all buffers used for generation of nuclear extracts from TSA-treated cells. Upon receiving the cells, they were thawed and washed once with ice-cold PBS and resuspended in five packed cell volumes (PCV) of HB1 (10 mM HEPES, pH 7.9, $10 \mathrm{mM}$ $\mathrm{KCl}, 0.1 \mathrm{mM}$ EDTA, $0.75 \mathrm{mM}$ spermidine, and $0.15 \mathrm{mM}$ spermine). After incubation on ice for 10 minutes, the cells were centrifuged and resuspended in two PCV HB1. The cells were subsequently lysed by Dounce homogenization. One-tenth volume of HB2 $(67.5 \%$ sucrose in HB1) was added, and the homogenates were centrifuged at $16,000 \times \mathrm{g}$ for 2 minutes. The nuclear pellets were resuspended in NLB (20 mM HEPES pH 7.6, $100 \mathrm{mM} \mathrm{KCl,} 12.5 \mathrm{mM} \mathrm{MgCl} 2,20 \%$ glycerol, $0.2 \mathrm{mM}$ EGTA, $0.2 \mathrm{mM}$ EDTA, and $2 \mathrm{mM}$ DTT) and rotated for 30 minutes. The nuclear lysates were centrifuged $(150,000 \times \mathrm{g}, 90$ minutes $)$, and the pellets were mixed with $0.33 \mathrm{~g}$ solid ammonium sulfate $/ \mathrm{ml}$ and resuspended in NLB. After rotation for 20 minutes, precipitated proteins were collected by centrifugation $(85,000 \times \mathrm{g}, 20$ minutes $)$ and resuspended gradually in approximately $1 \mathrm{ml}$ dialysis buffer (20 mM HEPES pH 7.6, $100 \mathrm{mM} \mathrm{KCl}, 12.5 \mathrm{mM} \mathrm{MgCl}$, $20 \%$ glycerol, $0.2 \mathrm{mM}$ EGTA, $0.2 \mathrm{mM}$ EDTA, and $2 \mathrm{mM}$ DTT). The extracts were dialyzed twice for $2 \mathrm{~h}$ in 1 liter of dialysis buffer. Supernatant was collected after centrifugation $\left(18,000 \times \mathrm{g}, 20\right.$ minutes), aliquoted, and stored at $-80^{\circ} \mathrm{C}$.

2.6. In Vitro Transcription Assay. The assay was performed as described by Kusk et al. [29] with minor modification. The ratio of nuclear extract protein to DNA was optimized for each set of nuclear extracts. In general, approximately $60 \mu \mathrm{g}$ extract protein was used with 1200-1500 ng DNA template per reaction. Briefly, nuclear extract was incubated at $30^{\circ} \mathrm{C}$ for 60 minutes with DNA template in a $30 \mu \mathrm{l}$ reaction mixture containing $20 \mathrm{mM}$ HEPES, pH7.9, $60 \mathrm{mM}$
$\mathrm{KCl}, 2 \mathrm{mM}$ DTT, $5 \mathrm{mM}$ creatine phosphate, $1 \mathrm{mM} \mathrm{3}$-OmethyGTP, $0.5 \mathrm{mM}$ ATP, $0.5 \mathrm{mM}$ CTP, $20 \mu \mathrm{M}$ UTP, $5 \mathrm{mM}$ $\mathrm{MgCl}_{2}, 10 \mu \mathrm{Ci}\left[\alpha{ }^{32} \mathrm{P}\right] \mathrm{UTP}(400 \mathrm{Ci} / \mathrm{mmol}), 10 \%$ glycerol, and $10 \mathrm{U}$ RNase $\mathrm{T} 1$. The reaction was terminated by addition of $300 \mu \mathrm{l}$ of Stop Solution (20 mM EDTA, $0.2 \mathrm{M} \mathrm{NaCl}, 1 \%$ SDS, and $10 \mu \mathrm{g}$ tRNA). RNA transcripts were extracted twice with phenol/chloroform/isoamyl alcohol followed by ethanol precipitation. The transcripts were resuspended in denaturing loading dye, incubated at $95^{\circ} \mathrm{C}$ for 3-4 minutes, and separated in 6\% denaturing polyacrylamide gels (Sequagel, National Diagnostics). Gels were dried and exposed to phosphorimaging screens. Radiolabeled transcripts were visualized with a Storm phosphoimager (Molecular Dynamics, Sunnyvale, CA).

For competition assays, nuclear extracts were preincubated with different amounts of the pAdML template for 30 minutes at $30^{\circ} \mathrm{C}$. Subsequently, the in vitro transcription reaction mixture containing NTPs and the MMTV template was added, and the transcription was allowed to proceed for 30 minutes at $30^{\circ} \mathrm{C}$. For analysis of de novo transcription, nuclear extracts were incubated with either MMTV or AdML template for 10 minutes at $30^{\circ} \mathrm{C}$ after which in vitro reaction mixture was added. Approximately 30 seconds later, water or Sarkosyl $(0.05 \%$ final concentration) was added, and transcription was allowed to proceed for $30 \mathrm{~min}$ before the reaction was stopped and transcript production was analyzed.

2.7. HDAC Assay. HDAC assays were carried out as described [17]. Nuclear extract protein $(20 \mu \mathrm{g})$ was diluted in assay buffer $(20 \mathrm{mM}$ Tris $\mathrm{HCl}, 150 \mathrm{mM} \mathrm{NaCl}, 0.5 \mathrm{mM}$ EDTA, and 5\% glycerol) in the presence or absence of TSA $(50 \mathrm{ng} / \mathrm{ml})$. The mixture was incubated for $30 \mathrm{~min}$ at $30^{\circ} \mathrm{C}$ to allow for HDAC inhibition. Subsequently, ${ }^{3} \mathrm{H}$-acetylated histones prepared from sodium butyrate-treated Hela cells were added, and incubation continued for an additional $30 \mathrm{~min}$. The reaction was stopped with denaturing buffer (1.44 M HCl and $0.24 \mathrm{M}$ acetic acid). Tritiated acetyl groups released by HDAC activity were extracted using ethyl acetate. Released cpms were measured by scintillation counting.

\section{Results}

3.1. HDAC Activity Is Required for Transcription from TATA Box Containing Core Promoters. Our previous study showed that the repression of MMTV transcription by HDAC inhibitors did not require sequences upstream of the TATA box [22]. Repression was conferred through a region of the promoter that extended from the TATA box to $100 \mathrm{bp}$ downstream of the transcription start site. In the current study, we further dissected the sequences required for repression. In particular, we were interested in determining the role of the core promoter, a region roughly defined as -40 to +40 bp relative to the start site of transcription (TSS). As reviewed in [30], the core promoter of a gene is made up of a series of elements which are not common to every core promoter but are generally found in subsets, depending on the particular gene. These include the upstream and 
downstream TFIIB recognition elements (BREs), the TATA box, and the initiator element (Inr), all of which are located upstream of the TSS with the exception of the Inr, which is located around the TSS. Some core promoters also contain elements downstream of the TSS including the downstream promoter element (DPE), the motif ten element (MTE), and the downstream core element (DCE).

The MMTV promoter contains a functional TATA box and initiator [31] but does not have BRE sequences. Downstream of the TSS there is a sequence that has some homology to the DPE consensus. To determine whether any of these elements confer repression mediated by HDAC inhibition, a series of luciferase reporter constructs were generated that contain various fragments of the MMTV promoter as shown in Figure 1(a). These constructs were transfected into Hela cells or 1470.2 cells, which were derived from the C127i mouse mammary adenocarcinoma cell line as described in Section 2. Consistent with our previous study, repression by TSA is not dependent on cisacting sequences upstream of the TATA box (compare pMluc with pMTV(TATA/+100)luc). Repression is also conferred by a fragment extending from the TATA box to the sequence resembling the DPE [pMTV(TATA-DPE)luc]. However, the DPE-like sequence is not required because a construct containing sequences between the TATA box and Inr [pMTV(TATA-Inr)luc] is sufficient to confer TSA-induced repression in both cell lines. Thus, we conclude that the repression of MMTV transcription mediated by HDAC inhibition is dependent solely on its functional core promoter elements.

In our previous study, we also showed that minimal promoter sequences from other viral and cellular genes were repressed by TSA treatment [22]. It is therefore possible that basal transcription of other core promoters is also sensitive to HDAC inhibition. Reporter constructs containing the analogous region from several viral and cellular TATA boxcontaining promoters were generated and transfected into Hela or 1470.2 cells. The results (Figure 2) show that TSA causes repression of core promoters from all genes tested, indicating that transcription dependent on the TATA to Inr region is generally sensitive to HDAC inhibition.

3.2. HDAC Activity Is Required for Transcriptional Initiation at the MMTV Promoter. The sensitivity of the MMTV core promoter to HDAC activity could be due to a role for HDACs in regulation of either transcriptional initiation or elongation. In the case of the former, the recruitment of RNA polymerase II (RNA pol II) may be impaired by HDAC inhibition. In the case of the latter, polymerase recruitment may be unaffected, but its ability to clear the $5^{\prime}$ transcribed region (first $100 \mathrm{bp}$ ) may be impaired due to pausing or stalling. To distinguish between these possibilities, we performed chromatin immunoprecipitation (ChIP) assays to measure the association of RNA pol II with the core promoter $/ 5^{\prime}$ transcribed region. If transcriptional initiation is impaired by HDAC inhibition, polymerase association with the core promoter may decrease due to a defect in polymerase recruitment. If HDAC activity is required to prevent pausing or stalling of polymerase, the association of RNA pol II with the $5^{\prime}$ transcribed region would be unchanged or somewhat increased as stalled polymerase accumulates. In the ChIP assays, we used an antibody that preferentially binds to RNA pol II with a hypophosphorylated C-terminal domain (CTD). This antibody has been shown to recognize both initiating as well as $5^{\prime}$ paused polymerase $[32,33]$ which does not contain a highly phosphorylated CTD because serine 2 phosphorylation has not yet occurred [34].

Cell line 1470.2 contains stably replicating copies of a transcription unit consisting of the full-length MMTV LTR/promoter driving the expression of the chloramphenicol acetyltransferase (CAT) reporter gene. Basal and activated expression of the MMTV-CAT gene in this cell line has been previously shown to be inhibited by TSA treatment [22]. Cells were treated with TSA for various times shown in Figure 3. As a positive control for RNA pol II association with the MMTV promoter, we included chromatin from cells treated with the synthetic glucocorticoid, Dexamethasone (Dex), which induces transcription from the promoter. As expected [35], Dex treatment rapidly but transiently induces recruitment of RNA pol II to the MMTV promoter (Figure 3(a), left panel). There is no association with downstream sequences in the CAT coding region (CAT CDS) that are well separated from the core promoter region, thus confirming that the antibody preferentially recognizes the polymerase in its initiating rather than its hyperphosphorylated elongating form.

In the presence of TSA, the association of RNA pol II with the core promoter $/ 5^{\prime}$ transcribed region clearly decreases over a time frame of 60 minutes (Figure 3(a), right panel). A quantitative representation of RNA pol II association data from 2 to 3 experiments with TSA is shown in Figure 3(b). By 60 minutes of TSA treatment, there is a $60 \%$ loss of polymerase. This is in good agreement with the magnitude of repression observed in the transfection experiments shown in Figure 1. In addition, both the kinetics and magnitude of TSA-induced decrease in promoter association of RNA pol II are highly consistent with changes in the rate of transcription from the MMTV promoter as measured by nuclear run-on in our previous study, in which we observed a 60-70\% drop in transcription from the MMTV-CAT gene within 30-60 minutes of TSA treatment [22]. From these data, we conclude that HDAC activity is required in some way for efficient RNA pol II recruitment and transcriptional initiation.

\subsection{Development of an In Vitro Transcription System to} Study the Role of HDACs in Transcriptional Initiation. Our previous study showed that HDAC inhibition does not lead to changes in chromatin structure, chromatin remodeling, or large increases in histone acetylation at the MMTV promoter [22], leading us to hypothesize that HDACs play a role in regulating the interactions or functions of nonhistone proteins essential for transcription from this promoter context. Thus, the mechanisms by which HDAC activity impact core promoter activity could be precisely defined by in vitro transcription assays, which allow a level of experimental control not possible in cell-based assays. To develop such an assay, we adopted the methods of Kusk et al. 


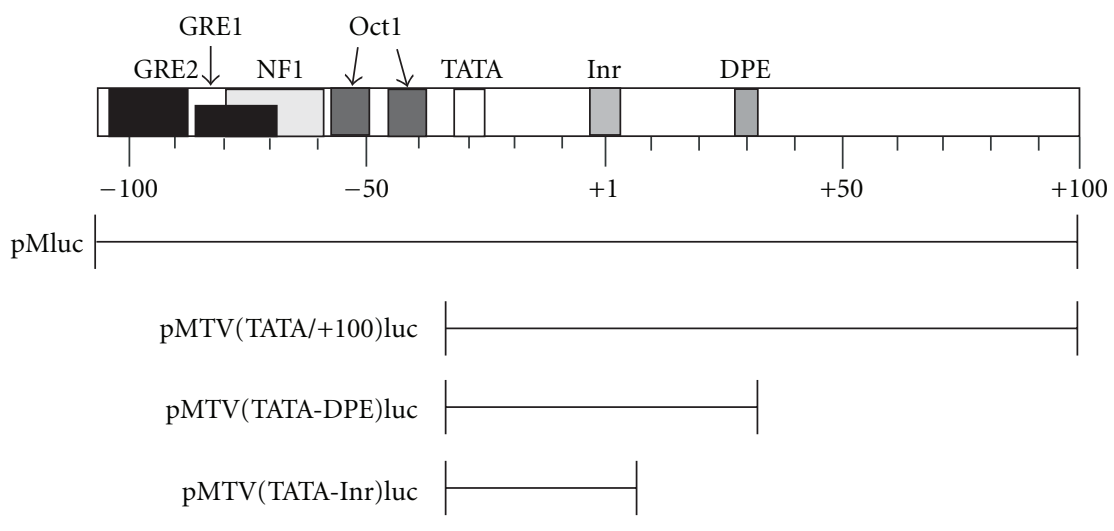

(a)

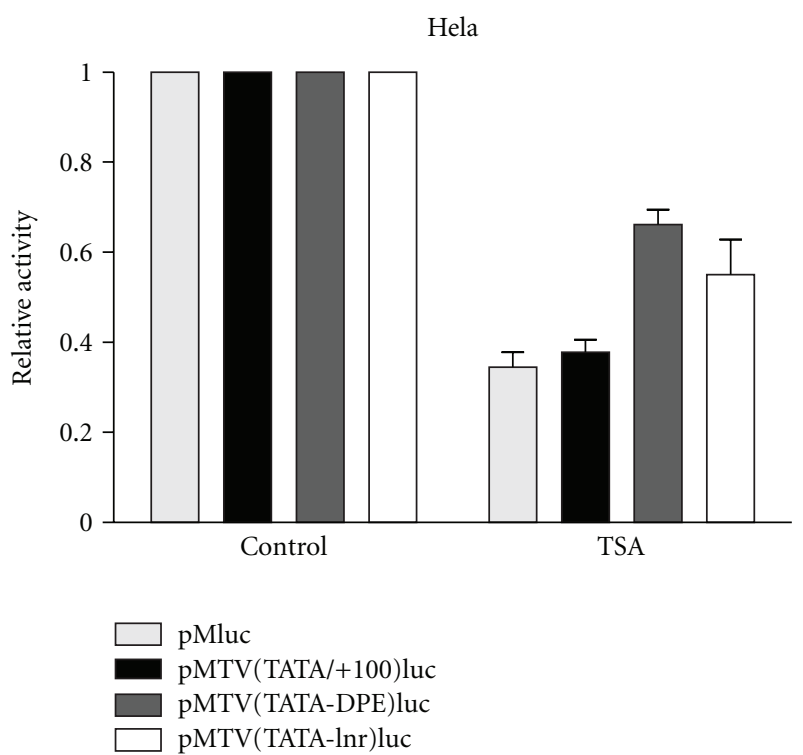

(b)

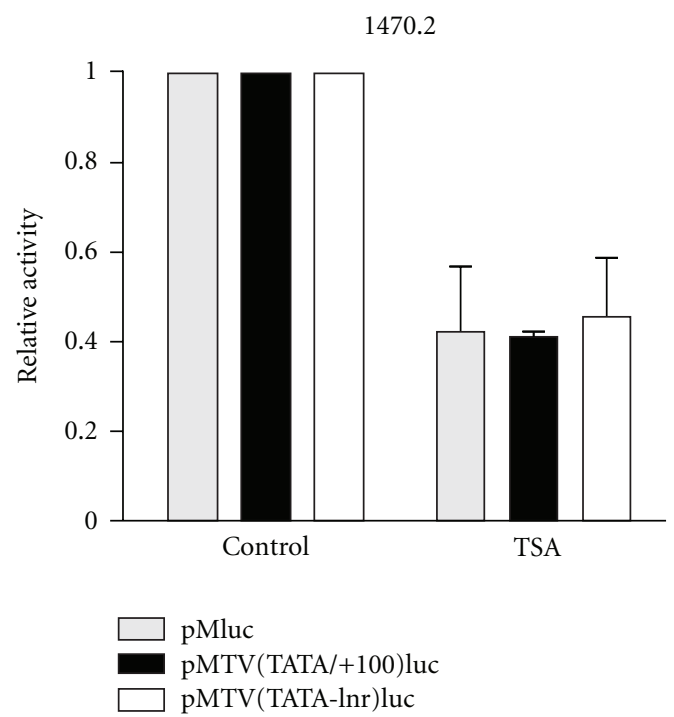

(c)

FIGURE 1: The MMTV core promoter confers transcriptional repression by the HDAC inhibitor, TSA. Various luciferase reporter constructs containing fragments of the MMTV promoter shown in (a) were transiently transfected into Hela or 1470.2 cells and treated with or without TSA $(50 \mathrm{ng} / \mathrm{ml})$ for $6 \mathrm{~h}$ prior to harvest. Cell lysates were assayed for luciferase activity, and these values were normalized to protein concentration for each sample. Normalized values for untreated (control) samples were set to 1, and values for treated samples were expressed as a fraction. Statistical analysis was performed on results from 3 to 7 independent experiments. Results from Hela and 1470.2 cells are shown in (b) and (c), respectively. Error bars represent SEM.

[29] who had measured MMTV core promoter function in vitro using templates with G-free cassettes [36]. Our goal was to compare transcription in nuclear extracts generated from cells treated with TSA to those generated from untreated cells to determine whether the repression observed in cellbased assays could be recapitulated in vitro. Because they can be easily grown in large quantities, we turned to Hela S3 cells to generate nuclear extracts by the strategy outlined in Figure 4(a). In the case of extracts made from cells treated with TSA, we maintained TSA in all buffers during extract preparation to prevent HDACs from becoming reactivated.

We chose to compare transcription of two promoters: MMTV and adenovirus major late (AdML). We designed an MMTV template containing the core promoter from the TATA box to the Inr upstream of a G-free cassette of approximately $400 \mathrm{bp}$ (Figure 4(b)). The AdML template contains a larger promoter fragment upstream of a smaller $\mathrm{G}$-free cassette. These templates were transcribed using equal quantities of nuclear extract from control (C) and TSAtreated (T) cells as shown in Figure 4(c). While transcription from the AdML template was similar in the two extracts, MMTV transcription was reduced in the extracts from TSA-treated cells. To eliminate the possibility that these results were due to differences in transcriptional competency specific to a particular set of extracts, we generated two additional sets of extracts (control and TSA-treated) and performed the same experiment. Figure 4(d) shows that the patterns of transcription from the two templates are very reproducible. MMTV transcription was consistently reduced in extracts from TSA-treated cells relative to a 


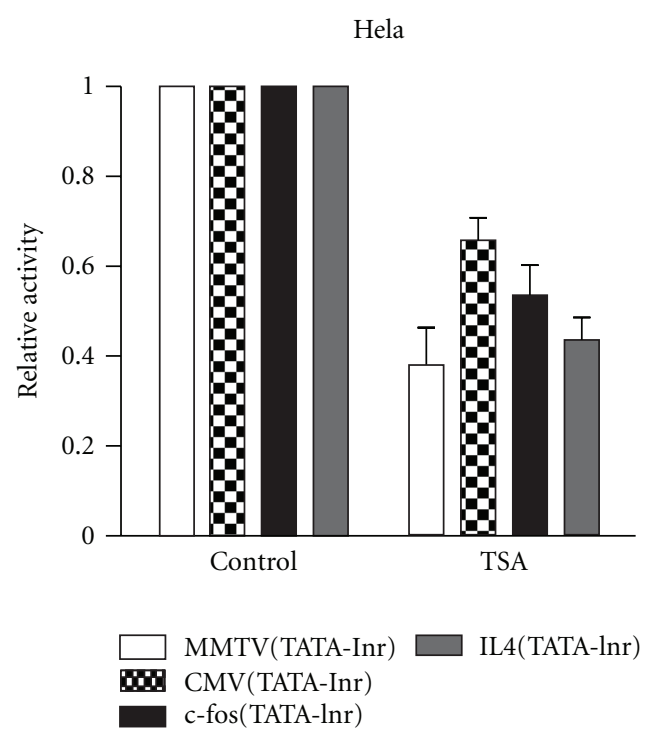

(a)

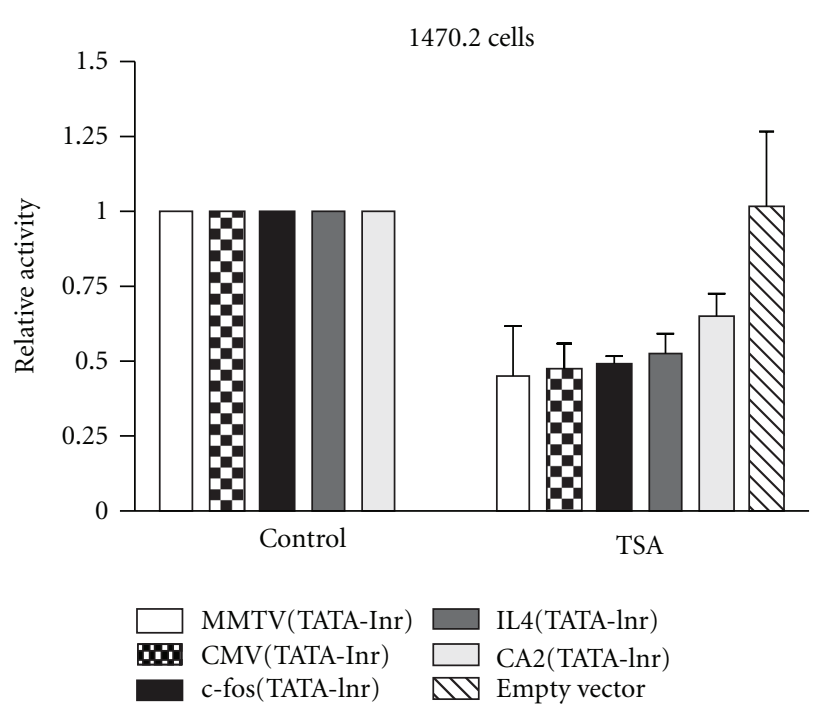

(b)

FIGURE 2: Transcription dependent on core promoters is repressed by TSA. Luciferase reporter constructs were generated which contain core promoters (TATA to Inr sequences) from several viral and cellular genes, including Cytomegalovirus (CMV), c-fos, interleukin 4 (IL4), and carbonic anhydrase II (CA2). These constructs were transiently transfected into either Hela or 1470.2 cells which were treated with or without TSA $(50 \mathrm{ng} / \mathrm{ml})$ for $6 \mathrm{~h}$. Cell lysates were assayed for luciferase activity, and the data was processed as described in the legend to Figure 1. Results from at least 3 independent experiments done with Hela or 1470.2 cells are shown. Error bars represent SEM.

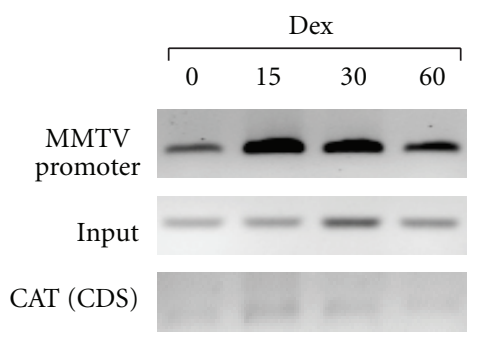

(a)

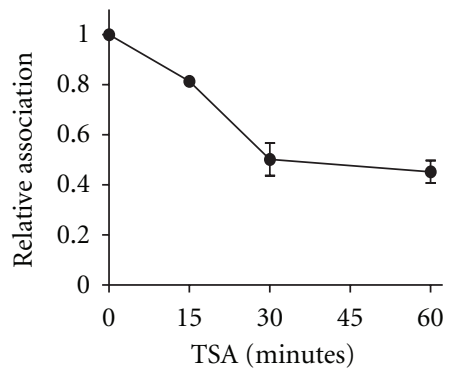

(b)

FIGURE 3: Inhibition of HDACs results in the loss of initiating RNA pol II from the MMTV promoter. 1470.2 cells were treated with either Dex $(100 \mathrm{nM})$ or TSA $(50 \mathrm{ng} / \mathrm{ml})$ for $0,15,30$, or 60 minutes, after which cells were processed for ChIP assay. Chromatin was immunoprecipitated with an RNA pol II antibody that recognizes the hypophosphorylated form of the CTD. Conventional PCR was performed on input and immunoprecipitated DNA using primers specific for the MMTV promoter and the coding region (CDS) of the CAT reporter. PCR results from a representative experiment are shown in (a). A graphical representation of the data from 2 to 3 independent experiments is shown in (b) for the TSA time course. Values from control samples were set to 1, and values from TSA-treated samples are expressed as a fraction. Error bars represent SEM.

matched control extract while AdML transcription was unaffected. We therefore conclude that the repression of MMTV transcription we observe in cell-based assays is recapitulated in vitro, and that this system can be utilized to ask precise mechanistic questions about the role of HDAC activity in transcriptional initiation.

\subsection{Transcriptional Reinitiation Rather Than De Novo} Transcription Is Sensitive to HDAC Inhibition. Transcriptional initiation is a broad term that incorporates two distinct processes: de novo transcription, which is the initial assembly of a preinitiation complex (PIC) and the first round of transcription, and reinitiation, which is the recruitment of RNA pol II to a pre-existing scaffold complex that is distinct in composition from the PIC. De novo transcription can be differentiated from reinitiation with the use of the detergent sarkosyl $[37,38]$. If added to a preassembled PIC prior to the addition of nucleotide triphosphates (NTPs), sarkosyl $(0.05 \%)$ will inhibit all transcription as shown for the AdML template in Figure 5(a). However, if it is added several seconds after NTP addition, the initial round of transcription will proceed and reinitiation is inhibited. As seen in Figure 5(a), this results in a significantly lower amount of transcript because only de novo transcription occurs. To determine whether HDAC activity is involved 


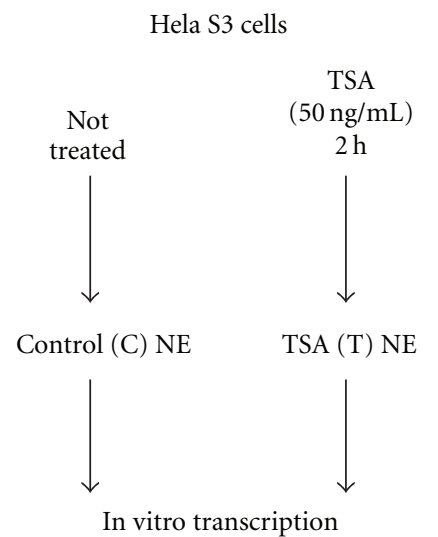

(a)

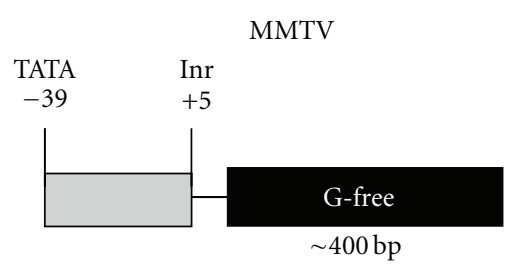

Adeno major late promoter

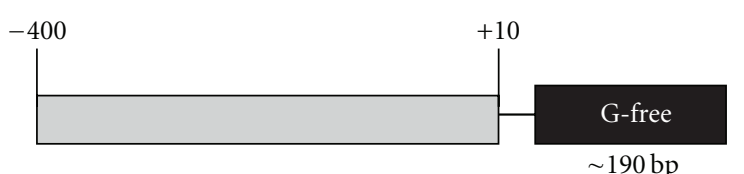

(b)

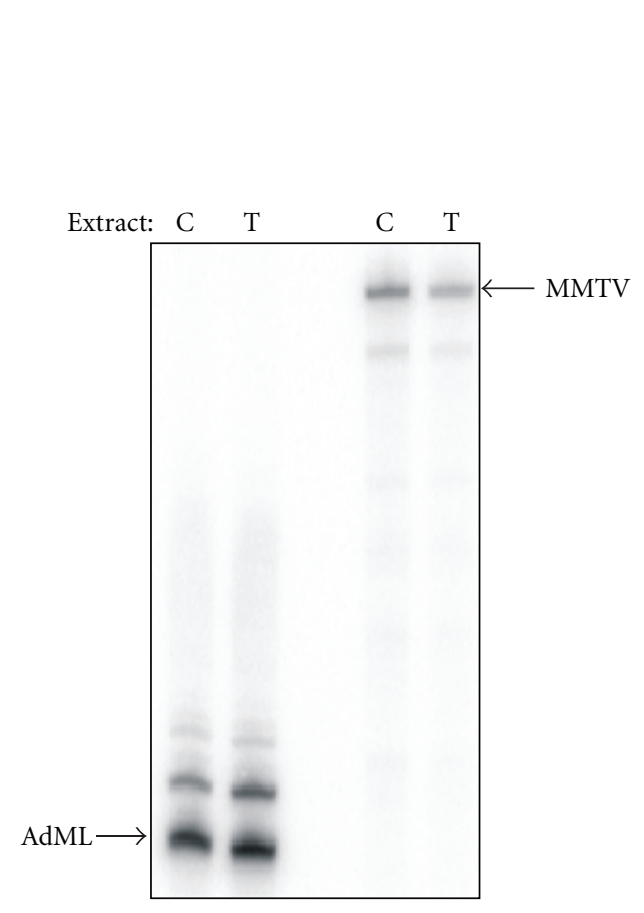

(c)

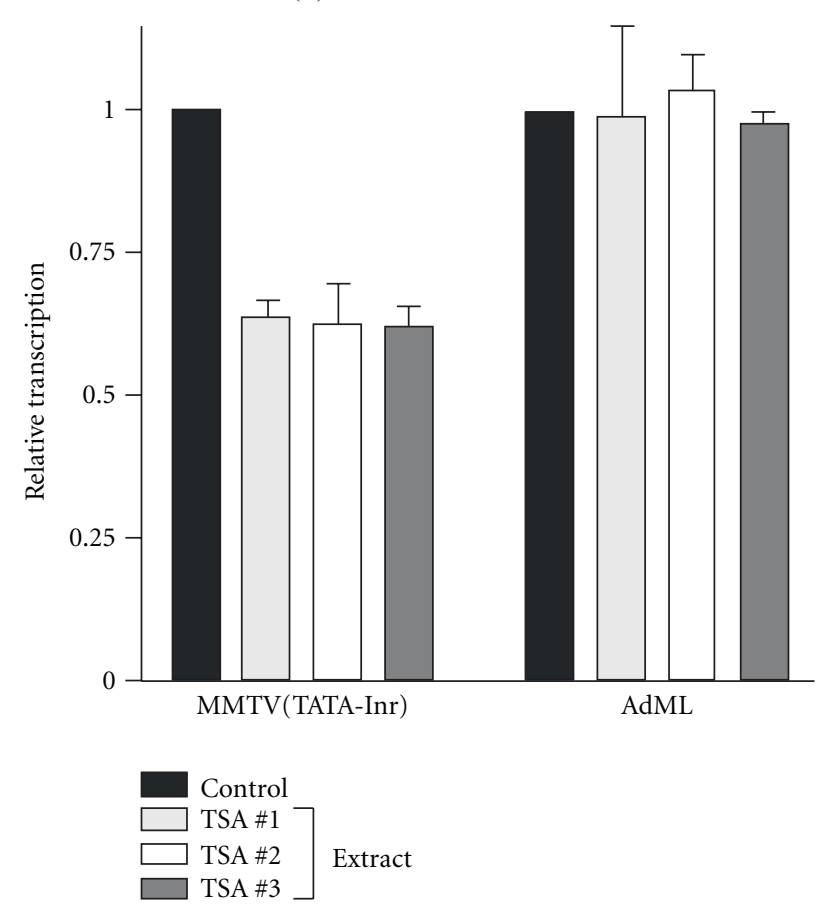

(d)

Figure 4: Repression of MMTV transcription by TSA is consistently recapitulated in an in vitro transcription assay. (a) Flow chart for a typical experiment. Nuclear extracts are generated from Hela S3 cells which were either untreated or TSA treated. These extracts were used for in vitro transcription assays with the templates shown in (b). The MMTV template contains the region from the TATA box to the initiator element and a G-free cassette of $400 \mathrm{bp}$. The adenovirus major late (AdML) template contains a promoter fragment from - $400 \mathrm{bp}$ to $+10 \mathrm{bp}$ with a G-free cassette of $190 \mathrm{bp}$. Results of a typical transcription assay are shown in (c). C-extract from untreated cells (Control). T-extract from TSA-treated cells. (d) shows a graphic representation of assay results using three independent sets of extracts. Each extract was assayed with each template at least three times. Transcription levels in the extracts from TSA-treated cells are expressed relative to those in the corresponding extracts from untreated (control) cells.

in regulation of these distinct initiation processes at the MMTV core promoter, we first established the extent to which reinitiation occurs in our in vitro transcription system. The MMTV or AdML templates were incubated with nuclear extracts from untreated cells to allow de novo assembly of the PIC. Nucleotide triphosphates (NTPs) were added to initiate transcription. After 30 seconds, sarkosyl or water was added and transcription was allowed to proceed for $1 \mathrm{~h}$. As shown in Figure 5(b), about 90\% of the transcription product from both templates is due to reinitiation since levels of transcript from each template in the presence of $0.05 \%$ sarkosyl were only $10 \%$ of those measured in its absence.

To determine whether HDAC inhibition impairs de novo transcription, the levels of MMTV transcript were measured after transcription in extracts from both control and TSA-treated cells in the presence or absence of sarkosyl. In Figure 5(c), transcript levels are expressed relative to those measured in control extracts in the absence of sarkosyl. As 


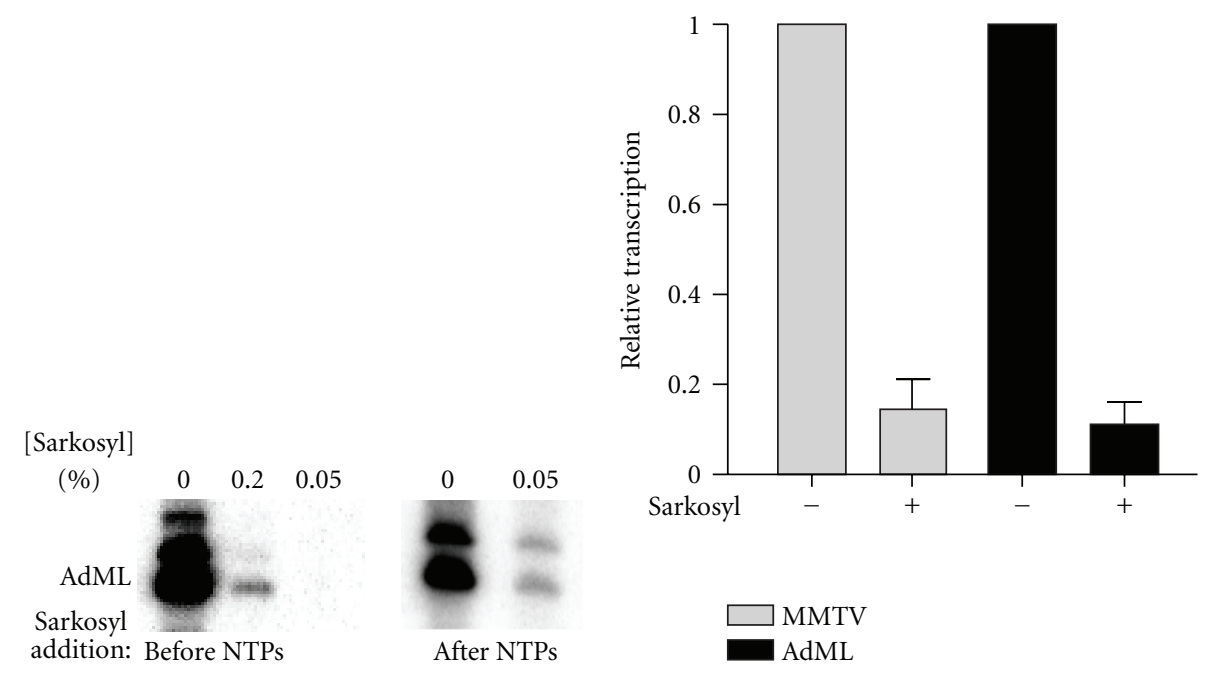

(a)

(b)

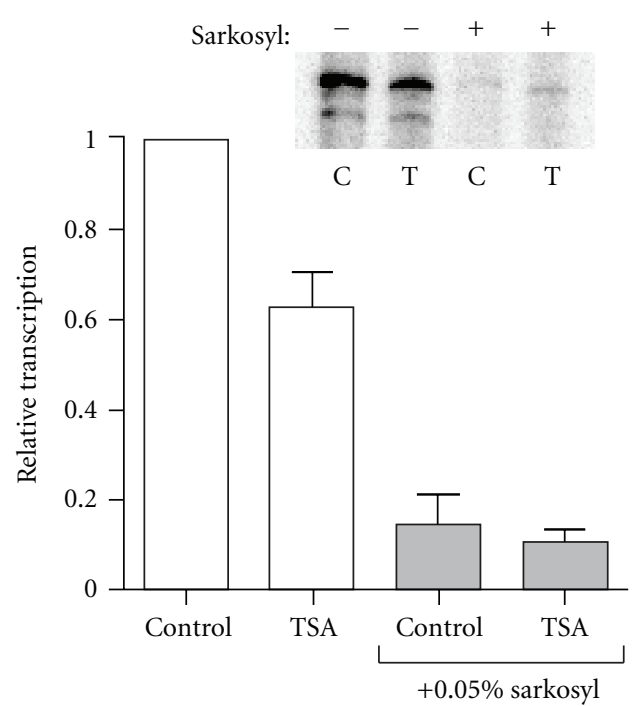

(c)

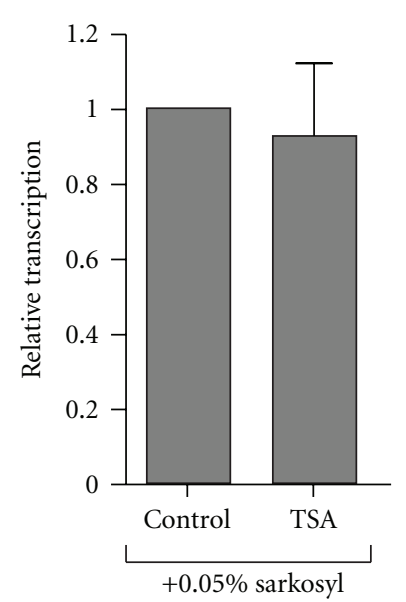

(d)

FIGURE 5: HDAC inhibition by TSA impairs reinitiation of transcription from the MMTV promoter in vitro. (a) In vitro transcription was carried out with the AdML template. Nuclear extracts were mixed with the template, and PIC assembly was allowed to proceed for $1 \mathrm{~h}$. Sarkosyl was added either 2 minutes before or 10 seconds after NTP addition. Transcription was allowed to proceed for 1 h. (b) In vitro transcription was carried out in the presence or absence of sarkosyl (0.05\%) added after NTP addition using either the MMTV or AdML templates as described in Section 2. Transcription levels in the presence of sarkosyl are expressed relative to levels of transcription measured in the absence of sarkosyl. (c, d) Transcription of the MMTV template was carried out using extracts from either control or TSA-treated cells in the presence or absence of sarkosyl $(0.05 \%)$. In (c), transcription levels are expressed relative to those measured in control extracts in the absence of sarkosyl. In (d), transcription levels measured in extracts from TSA-treated cells are expressed relative to those measured in control extracts, both in the presence of sarkosyl. The results shown were obtained from three independent experiments, and error bars represent SEM.

expected, transcription of the MMTV template is reduced in extracts from TSA-treated cells in the absence of sarkosyl. However, in the presence of sarkosyl, transcript levels are similar in the two extracts. In Figure 5(d), transcript levels produced in the extracts from TSA-treated cells in the presence of sarkosyl are expressed relative to those measured in the control extracts under the same conditions to confirm that there is no significant difference. These results show that de novo transcription is unaffected by the lack of HDAC activity and strongly suggest that the reduction in MMTV transcription in the extracts derived from TSA-treated cells is due to impaired reinitiation.

\subsection{Initiation Complex Stability and HDAC Inhibition.} Two potential mechanisms by which HDAC activity might regulate transcriptional reinitiation include (1) the destabilization and dissociation of the reinitiation scaffold in the absence of HDAC activity and (2) inhibition of the activity of the scaffold and/or its ability to recruit RNA pol II. To distinguish between these possibilities, we performed 


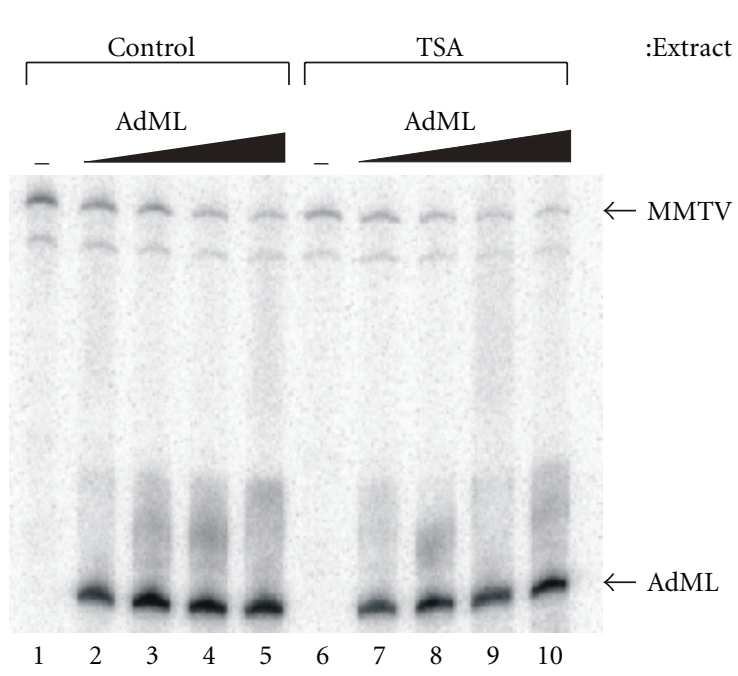

(a)

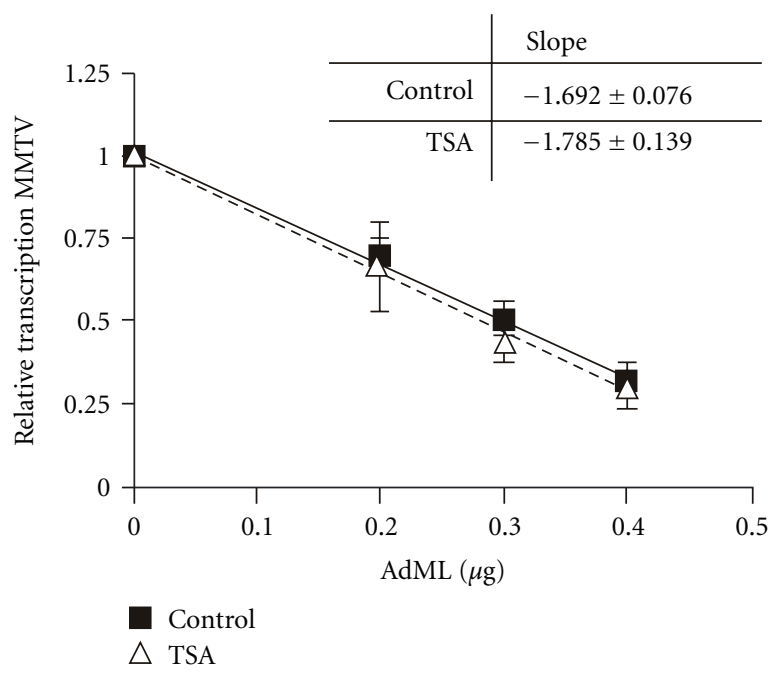

(b)

FIgURE 6: HDAC inhibition does not affect the stability of initiation complexes at the MMTV promoter as determined by template competition assay. Transcription of the MMTV template in extracts from control or TSA-treated cells was carried out in the presence of increasing amounts of AdML template. A representative experiment is shown in (a). (b) shows the relative level of MMTV transcription in extracts from either control or TSA-treated cells. Transcriptional levels in each extract are expressed relative to those measured in the absence of AdML template. The results shown are derived from at least 3 independent experi

ments. The data were subjected to linear regression analysis to generate lines and slopes using GraphPad Prism. Error bars represent SEM.

template competition assays in which transcription from a fixed amount of MMTV template was carried out in the presence of increasing amounts of the AdML template as a competitor. As the amount of AdML DNA increases, transcription from the MMTV promoter should decrease. This can be observed in Figure 6(a), lanes 1-5, and lanes 6-10 for transcription carried out in extracts from control and TSA-treated cells, respectively. The relationship between MMTV transcript levels and AdML template for each extract was then analyzed by linear regression to generate a line as shown graphically in Figure 6(b). If the lack of HDAC activity results in major destabilization of the reinitiation scaffold, the slope of the line depicting transcript levels in the extracts from TSA-treated cells would be significantly higher (steeper line) because the AdML template would compete more effectively for factors. However, the data shows that the slopes of the two lines are not significantly different even though the level of overall MMTV transcription in the extracts from TSA-treated cells is lower (shown in Figure 6(a)). This result suggests that the loss of HDAC activity does not affect the overall stability of the reinitiation scaffold but rather decreases its activity.

\subsection{HDAC Activity Is Not Required during the Transcription} Process. HDAC activity might regulate the efficiency of reinitiation either by being directly required during the process of transcriptional initiation or by regulating the activity of components of the reinitiation scaffold. In the case of the former, a critical deacetylation reaction may take place with every round of initiation, analogous to the way the CTD of RNA pol II must be dephosphorylated before it can reinitiate transcription. Alternatively, HDACs regulate the activity of a scaffold component, perhaps through its acetylation status. Treatment of cells with HDAC inhibitors prior to generation of nuclear extracts would lead to the accumulation of this component in its functionally impaired state. This accumulation would thereby increase the chances of assembling functionally impaired scaffold complexes.

To distinguish between these possibilities, we asked whether TSA added to extracts from untreated control cells could inhibit MMTV transcription according to the scheme outlined in Figure 7(a). If HDAC action was required during each round of transcription, we expect that transcription would be inhibited by the addition of TSA to the control extract. To establish that the added TSA inhibits HDAC activity, an HDAC assay was performed to compare control extracts to which either TSA or DMSO had been added. As shown in Figure 7(b), HDAC activity is significantly inhibited by addition of TSA to a level even lower than that measured in extracts generated from cells treated with TSA. In vitro transcription was carried out on the MMTV templates using either extracts generated from cells treated with or without TSA or with the control extract to which TSA or the vehicle, DMSO, had been added. Consistent with previous experiments, MMTV transcription was reduced in extract from cells treated with TSA relative to control extracts. However, the addition of TSA directly to the control extract did not impair MMTV transcription. This result indicates that HDAC activity is not directly required during the initiation and elongation process and suggests that impaired function of the assembled basal transcription machinery is responsible for the reduced rate of transcriptional initiation. 


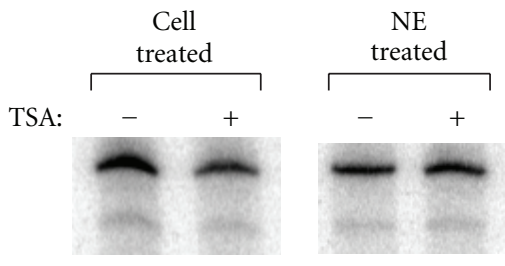

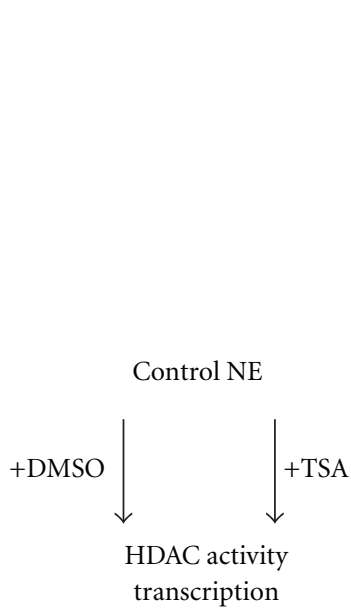

(a)

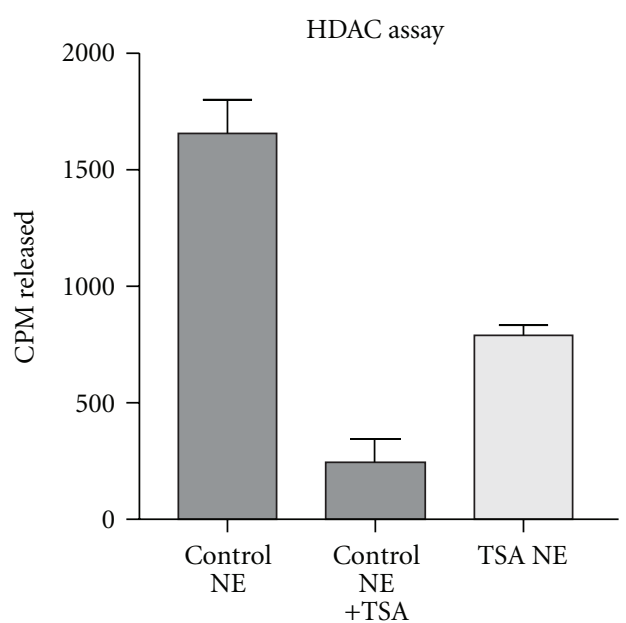

(b)

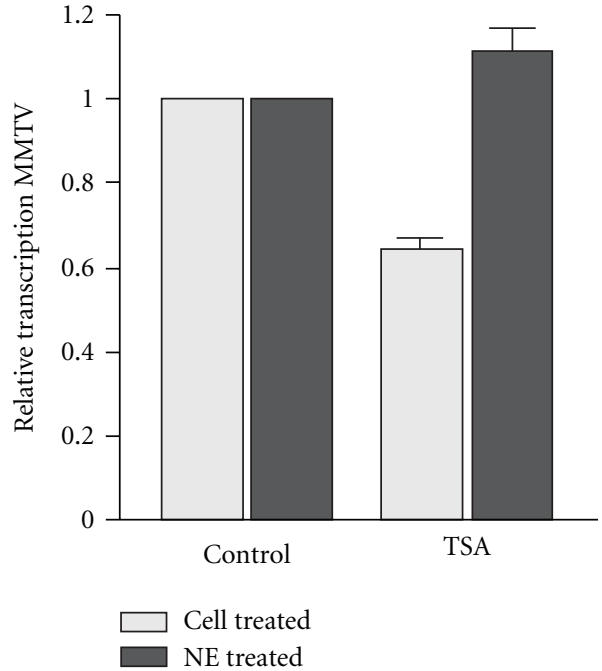

(c)

FIGURE 7: HDAC activity is not required during transcription of the MMTV promoter. (a) shows the experimental outline. Vehicle (DMSO) or TSA $(50 \mathrm{ng} / \mathrm{ml})$ was added to extracts from untreated (control) cells. These extracts were then subjected to HDAC assay or used for in vitro transcription. (b) shows the results of HDAC assays on control extracts to which vehicle or TSA had been added. The same assay was performed on extracts from cells treated with TSA as a comparison. The graph represents data from 3 independent experiments. (c) shows the results of in vitro transcription from the MMTV template comparing extracts from cells treated with or without TSA (Cell treated) with extracts from untreated (control) cells to which vehicle or TSA had been added (NE treated). The graph was generated from data derived from at least 3 independent experiments. Error bars represent SEM.

\section{Discussion}

Despite accumulating evidence that HDACs and protein deacetylation play positive roles in regulation of transcription, virtually nothing is known about the underlying mechanisms. In our previous study, we established that inhibition of HDAC activity caused repression of MMTV promoter activity that was transcriptional in nature and independent of changes in histone acetylation, chromatin structure, chromatin remodeling, and the binding of transcription factors upstream from the TATA box. In the current study, we have investigated the mechanisms behind this repression and found that it is mediated through the functional core promoter elements of the MMTV LTR and can also be observed with the core promoters of other genes. In addition, this repression is due to the inhibition of transcriptional initiation and correlates with decreased association of RNA pol II with the promoter. Development of an in vitro transcription system that recapitulates repression due to HDAC inhibition has allowed us to determine that reinitiation rather than de novo transcriptional initiation is sensitive to HDAC inhibition. The impairment of reinitiation is not due to wholesale destabilization of the scaffold complex or a requirement for HDAC activity during each round of initiation, but rather, the results indicate that the ability of the assembled scaffold to recruit RNA pol II is inhibited. This study extends our knowledge of the transcriptional roles of HDACs and provides novel insights into the role of HDACs in facilitating transcription through the core promoter and basal transcription machinery.

Analysis of MMTV sequences necessary to confer repression on transcription through HDAC inhibition revealed its surprising dependence on the core promoter. The analogous region from several other genes displayed similar behavior. Interestingly, repression of both promoters of the c-src gene by HDAC inhibitors was found to be mediated through their core promoter regions $[39,40]$. The core promoter is often viewed, not as an active participant in gene regulation, but only as a place to assemble the basal transcription machinery in response to the action of transcription factors bound to the promoter or distant enhancers. However, core promoter elements are not uniform between genes but usually occur in subsets (e.g., TATA/Inr, Inr/DPE, BRE/TATA/DPE) (reviewed in [30]). In addition, some transcriptional activators work more efficiently with a particular core promoter subtype, such as Caudal, a key regulator of Hox genes, which 


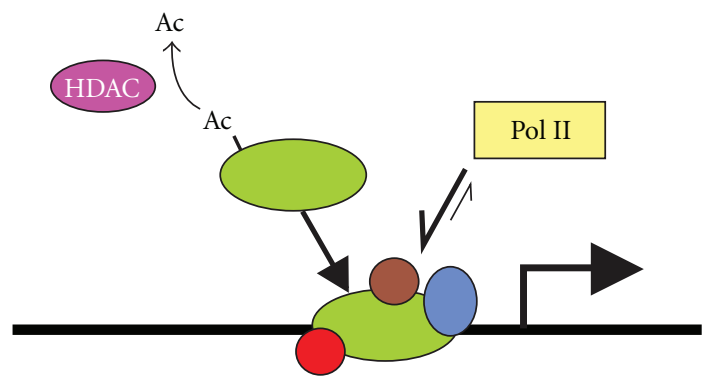

(a)

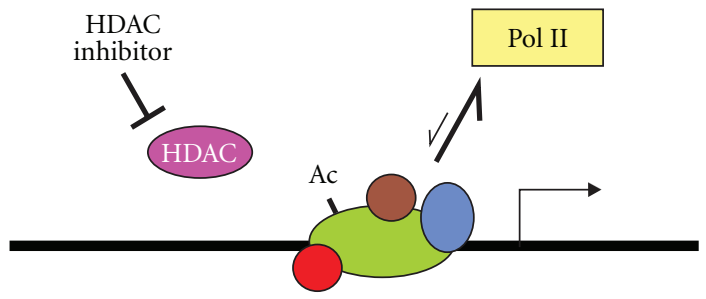

(b)

Figure 8: Proposed model for the role of HDACs in basal transcription from the MMTV promoter. (a) HDACs remove acetyl groups from proteins that are part of the reinitiation scaffold. This may occur prior to binding to the template (as shown) or at the template. This deacetylation facilitates binding of RNA pol II and the initiation of transcription. When HDAC activity is inhibited through HDAC inhibitors, shown in (b) persistent acetylation of basal transcription machinery components does not destabilize the complex or prevent its assembly. However, the recruitment of RNA pol II is impaired, leading to a decrease in the rate of transcriptional initiation.

prefers core promoters with a DPE to those with a TATA box $[41,42]$. Such examples imply that the basal transcription complexes that form at these different core promoters differ somewhat in configuration, stability, or composition and therefore may respond distinctly to regulatory signals.

All the core promoters tested in our study contain both a TATA box and Inr element; some also contain an upstream BRE (CMV and CA2). Our results would seem to imply that all genes with TATA/Inr-containing core promoters are repressed by HDAC inhibitors, but we showed this not to be the case in our previous study [22]. We suggest that when transcription is strictly dependent on this type of core promoter, there is a requirement for HDAC activity. However, in the context of the other regulatory regions that control the expression of a particular gene, the combination of transcriptional regulators that bind to them can overcome this requirement. For promoters such as MMTV, the transcriptional regulators that bind upstream of the TATA box are unable to overcome the repression of basal transcription caused by the loss of HDAC activity. The gene specificity of the core promoter requirement for HDAC activity may be mediated by dependence of key transcriptional regulators on different components of the basal transcription complexes to facilitate transcriptional initiation. Some subunits of the Mediator and TFIID complexes have been shown to function in a gene-specific fashion and/or to be differentially utilized by promoter-bound transcription factors (reviewed in [43, 44]). For example, the ligand-dependent nuclear receptors have been shown to require the presence of the Med1 subunit of the mediator complex to activate some target promoters, and the estrogen receptor (ER) has been shown to contact TAF10 of TFIID to activate transcription [45]. Interestingly, GR has been reported to require different mediator subunits in distinct promoter contexts [46, 47]. Posttranslational modification (such as acetylation) of complex subunits required for interaction with particular transcription factors may disrupt those interactions and lead to a reduction in activated or basal transcription. Alternatively, a transcription factor bound to a gene may bypass the negative effects of a functionally impaired subunit of the basal transcription machinery if it requires other subunits to facilitate transcription. In the case of MMTV, we propose that the upstream factors that drive transcription require the function of the impaired subunit(s) that causes repression of core promoter activity and therefore cannot overcome the loss of HDAC activity.

Our experiments with sarkosyl show that de novo transcriptional initiation from the MMTV promoter is unaffected by HDAC inhibition, indicating that the repression observed is due to impaired reinitiation. De novo initiation involves the assembly of the PIC at a completely inactivate promoter. Once the original round of transcription has begun and the polymerase leaves the core promoter, a scaffold is left behind which contains general transcription factors TFIID, -IIA, -IIH, -IIE, and the mediator complex [48]. Some transcription factors stabilize this scaffold and facilitate transcription by increasing the rate of reinitiation [48-51]. In a study of the effects of TSA on HIV transcription in vitro, it was observed that TSA increased the amount of de novo transcription [51]. These experiments were performed with chromatin-assembled HIV templates, and the stimulatory effect of TSA on de novo transcription may have been due to increased histone acetylation, which has been shown to facilitate interactions between transcription factors and chromatin (reviewed in [52]). The same study also showed that TSA had little effect on reinitiation of HIV transcription [51]. However, it was also determined that the transcription factors which bind the HIV promoter were highly efficient in stimulating reinitiation in vitro. As we discussed above, it is certainly possible that this combination of factors can overcome any negative effects of acetylation on reinitiation in the HIV promoter context. Our studies of the MMTV promoter strongly suggest that this is not true of the combination of transcription factors that control its transcriptional activity. However, the specific effects of HDAC inhibition on reinitiation versus de novo initiation provides a potential explanation for the ability of GR to partially activate the MMTV promoter in the presence of TSA or HDAC1 depletion $[17,22]$. There is a level of basal MMTV transcription in our cell lines, which is likely to be due to either stochastic de novo assembly of the PIC and/or a low rate of reinitiation from pre-existing scaffold complexes. GR may act to both promote de novo assembly of the PIC at MMTV templates within a cell population and increase reinitiation at preassembled scaffold complexes. Our results 
suggest that GR-induced de novo transcription within a cell population would not be affected by loss of HDAC activity but that reinitiation would be impaired, thus decreasing the magnitude of GR-activated MMTV transcription but not completely inhibiting it.

Why would HDAC activity and deacetylation affect only reinitiation and not de novo transcription? A recent study indicates that the structure of the reinitiation scaffold is distinct from that of the initial PIC. Knuesel and colleagues showed that the Cdk8 submodule of the mediator can bind to the reinitiation scaffold but not the initial PIC [53]. Once bound, it can repress the rate of reinitiation because RNA pol II cannot rebind. They hypothesize that there is a structural shift in mediator structure that occurs once pol II has cleared the initial PIC which allows this module to bind and act as a rheostat. It is also possible that conformational changes occur in other components of the basal transcription machinery after the initial round of transcription. A difference between the initial PIC and the reinitiation scaffold may also explain the differential ability of transcription factors to facilitate reinitiation [48]. Our study has elucidated another key difference between reinitiation and de novo transcription and that is a requirement for HDAC activity.

How does HDAC activity affect reinitiation? The template competition experiments indicate that HDAC activity does not stabilize the reinitiation scaffold since the ability of the AdML template to compete with MMTV for complex assembly was not increased in extracts from TSA-treated cells. This finding implies that it is the activity rather than the overall stability of the scaffold that is impaired when HDAC activity is compromised. The ChIP experiments showed that TSA treatment resulted in decreased association of RNA pol II with the MMTV promoter. Thus, it is possible that the ability of the reinitiation scaffold to recruit pol II is dependent on HDAC activity as shown in the model depicted in Figure 8. However, inhibition of HDACs through addition of TSA to the control nuclear extracts did not lead to inhibition of MMTV transcription, meaning that HDAC activity is not required during the process of reinitiation, perhaps removing acetyl groups that were acquired during initiation and must be removed prior to binding of another pol II complex, analogous to the phosphorylation cycles of the pol II CTD [34].

We hypothesize that treatment of cells with TSA prior to generation of the nuclear extracts led to changes in the acetylation status of one or several proteins that regulate transcriptional initiation. This regulation could be indirect, involving HDAC target proteins that influence the activity or post-translational modification of the basal transcription machinery but are not directly involved in transcriptional initiation at the core promoter. However, we argue that the effect of acetylation on transcriptional initiation is more direct. First, nuclear run-on experiments in our previous study clearly showed that basal transcription from the MMTV promoter decreases immediately, within 5 minutes of TSA treatment [22]. A delay might be expected if an indirect mechanism was in play. Second, it has been shown that HDAC1 is constitutively present at the MMTV promoter and that its depletion by RNA interference impairs MMTV transcription [17]. A change in its activity by exposure to HDAC inhibitors could immediately affect the dynamics of acetylation at the promoter to cause a rapid decrease in transcription through increased acetylation of proteins at the promoter. These proteins could be subunits of the complexes that make up the reinitiation scaffold or proteins known to directly regulate basal transcription such as NC2, BTAF, or the Cdk8 module of the mediator complex (reviewed in [43, $54]$ ). Loss of HDAC activity would increase the acetylated fraction of these proteins, thereby increasing the chance that acetylated forms were incorporated into PICs and scaffolds formed at our templates in vitro. HAT activity in the nuclear extracts may not have been robust enough or targeted enough to significantly increase acetylation of these proteins when HDACs were inhibited in vitro (Figure 7). In spite of the fact that TFIID contains an acetyltransferase (TAF1) and robust HATs such as CBP, p300, and PCAF are recruited to many promoters, very little is known about acetylation of the basal transcription machinery, including whether there are acetylated subunits, and if so, how acetylation affects their function.

In summary, our study has uncovered a novel mechanism by which HDACs function in facilitating transcriptional initiation. Future studies will be directed at identifying the critical target of HDAC activity that regulates basal transcription and further dissection of the mechanism by which HDAC activity impacts reinitiation.

\section{Acknowledgments}

The authors are grateful to Dr. Gordon Hager (NIH) and Dr. Robert Roeder (Rockefeller University) for the gift of Gfree plasmids and Dr. Yi Qiu (NIH) for tritiated histones and assistance in carrying out HDAC assays. They thank Dr. W. Lee Kraus (UT-Southwestern) for technical advice in performing the various in vitro transcription assays. They also appreciate the efforts of Ms. Jamie Jackson (University of Arizona) in performing transfection and reporter assays. Finally, they gratefully acknowledge Dr. Kevin Gardner (NIH) for a critical reading of the paper. This work was supported by the intramural research program of the National Cancer Institute and startup funds from the University of Arizona to C. L. Smith.

\section{References}

[1] M. Haberland, R. L. Montgomery, and E. N. Olson, "The many roles of histone deacetylases in development and physiology: implications for disease and therapy," Nature Reviews Genetics, vol. 10, no. 1, pp. 32-42, 2009.

[2] S. Balasubramanian, E. Verner, and J. J. Buggy, "Isoformspecific histone deacetylase inhibitors: the next step?" Cancer Letters, vol. 280, no. 2, pp. 211-221, 2009.

[3] C. Colussi, B. Illi, J. Rosati et al., "Histone deacetylase inhibitors: keeping momentum for neuromuscular and cardiovascular diseases treatment," Pharmacological Research, vol. 62, no. 1, pp. 3-10, 2010. 
[4] M. A. Halili, M. R. Andrews, M. J. Sweet, and D. P. Fairlie, "Histone deacetylase inhibitors in inflammatory disease," Current Topics in Medicinal Chemistry, vol. 9, no. 3, pp. 309 319, 2009.

[5] N. L. Wiech, J. F. Fisher, P. Helquist, and O. Wiest, "Inhibition of histone deacetylases: a pharmacological approach to the treatment of non-cancer disorders," Current Topics in Medicinal Chemistry, vol. 9, no. 3, pp. 257-271, 2009.

[6] V. M. Richon, J. Garcia-Vargas, and J. S. Hardwick, "Development of vorinostat: current applications and future perspectives for cancer therapy," Cancer Letters, vol. 280, no. 2, pp. 201-210, 2009.

[7] W. Fischle, Y. Wang, and C. D. Allis, "Histone and chromatin cross-talk," Current Opinion in Cell Biology, vol. 15, no. 2, pp. 172-183, 2003.

[8] K. B. Glaser, M. J. Staver, J. F. Waring, J. Stender, R. G. Ulrich, and S. K. Davidsen, "Gene expression profiling of multiple histone deacetylase (HDAC) inhibitors: defining a common gene set produced by HDAC inhibition in T24 and MDA carcinoma cell lines," Molecular Cancer Therapeutics, vol. 2, no. 2, pp. 151-163, 2003.

[9] C. Van Lint, S. Emiliani, and E. Verdin, "The expression of a small fraction of cellular genes is changed in response to histone hyperacetylation," Gene Expression, vol. 5, no. 4-5, pp. 245-253, 1996.

[10] C. Foglietti, G. Filocamo, E. Cundari et al., "Dissecting the biological functions of Drosophila histone deacetylases by RNA interference and transcriptional profiling," Journal of Biological Chemistry, vol. 281, no. 26, pp. 17968-17976, 2006.

[11] J. M. A. Moreira, P. Scheipers, and P. Sørensen, "The histone deacetylase inhibitor Trichostatin A modulates CD4+ T cell responses," BMC Cancer, vol. 3, article 30, 2003.

[12] G. Reid, R. Métivier, C. Y. Lin et al., "Multiple mechanisms induce transcriptional silencing of a subset of genes, including oestrogen receptor $\alpha$, in response to deacetylase inhibition by valproic acid and trichostatin A," Oncogene, vol. 24, no. 31, pp. 4894-4907, 2005.

[13] O. H. Krämer, M. Göttlicher, and T. Heinzel, "Histone deacetylase as a therapeutic target," Trends in Endocrinology and Metabolism, vol. 12, no. 7, pp. 294-300, 2001.

[14] M. J. Peart, G. K. Smyth, R. K. van Laar et al., "Identification and functional significance of genes regulated by structurally different histone deacetylase inhibitors," Proceedings of the National Academy of Sciences of the United States of America, vol. 102, no. 10, pp. 3697-3702, 2005.

[15] C. L. Smith, "A shifting paradigm: histone deacetylases and transcriptional activation," BioEssays, vol. 30, no. 1, pp. 15-24, 2008.

[16] I. Nusinzon and C. M. Horvath, "Histone deacetylases as transcriptional activators? Role reversal in inducible gene regulation," Science's STKE, vol. 2005, no. 296, article re11, 2005.

[17] Y. Qiu, Y. Zhao, M. Becker et al., "HDAC1 acetylation is linked to progressive modulation of steroid receptor-induced gene transcription," Molecular Cell, vol. 22, no. 5, pp. 669-679, 2006.

[18] I. Nusinzon and C. M. Horvath, "Interferon-stimulated transcription and innate antiviral immunity require deacetylase activity and histone deacetylase 1," Proceedings of the National Academy of Sciences of the United States of America, vol. 100, no. 25, pp. 14742-14747, 2003.

[19] H. M. Chang, M. Paulson, M. Holko et al., "Induction of interferon-stimulated gene expression and antiviral responses require protein deacetylase activity," Proceedings of the National Academy of Sciences of the United States of America, vol. 101, no. 26, pp. 9578-9583, 2004.

[20] M. Xu, L. Nie, S. H. Kim, and X. H. Sun, "STAT5-induced Id-1 transcription involves recruitment of HDAC1 and deacetylation of C/EBP $\beta$," EMBO Journal, vol. 22, no. 4, pp. 893-904, 2003.

[21] Z. Wang, C. Zang, K. Cui et al., "Genome-wide mapping of HATs and HDACs reveals distinct functions in active and inactive genes," Cell, vol. 138, no. 5, pp. 1019-1031, 2009.

[22] N. M. Mulholland, E. Soeth, and C. L. Smith, "Inhibition of MMTV transcription by HDAC inhibitors occurs independent of changes in chromatin remodeling and increased histone acetylation," Oncogene, vol. 22, no. 31, pp. 4807-4818, 2003.

[23] E. H. Bresnick, S. John, D. S. Berard, P. LeFebvre, and G. L. Hager, "Glucocorticoid receptor-dependent disruption of a specific nucleosome on the mouse mammary tumor virus promoter is prevented by sodium butyrate," Proceedings of the National Academy of Sciences of the United States of America, vol. 87, no. 10, pp. 3977-3981, 1990.

[24] J. Bartsch, M. Truss, J. Bode, and M. Beato, "Moderate increase in histone acetylation activates the mouse mammary tumor virus promoter and remodels its nucleosome structure," Proceedings of the National Academy of Sciences of the United States of America, vol. 93, no. 20, pp. 10741-10746, 1996.

[25] J. R. Lambert and S. K. Nordeen, "Steroid-selective initiation of chromatin remodeling and transcriptional activation of the mouse mammary tumor virus promoter is controlled by the site of promoter integration," Journal of Biological Chemistry, vol. 273, no. 49, pp. 32708-32714, 1998.

[26] M. A. Wilson, A. R. Ricci, B. J. Deroo, and T. K. Archer, "The histone deacetylase inhibitor trichostatin A blocks progesterone receptor-mediated transactivation of the mouse mammary tumor virus promoter in vivo," Journal of Biological Chemistry, vol. 277, no. 17, pp. 15171-15181, 2002.

[27] S. K. Nordeen, "Luciferase reporter gene vectors for analysis of promoters and enhancers," BioTechniques, vol. 6, no. 5, pp. 454-458, 1988.

[28] A. Magklara and C. L. Smith, "A composite intronic element directs dynamic binding of the progesterone receptor and GATA-2," Molecular Endocrinology, vol. 23, no. 1, pp. 61-73, 2009.

[29] P. Kusk, K. E. Carlson, B. S. Warren, and G. L. Hager, "Role of the TATA box in transcription of the mouse mammary tumor virus long terminal repeat," Molecular Endocrinology, vol. 9, no. 9, pp. 1180-1192, 1995.

[30] T. Juven-Gershon and J. T. Kadonaga, "Regulation of gene expression via the core promoter and the basal transcriptional machinery," Developmental Biology, vol. 339, no. 2, pp. 225229, 2010.

[31] J. Pierce, B. E. Fee, M. G. Toohey, and D. O. Peterson, "A mouse mammary tumor virus promoter element near the transcription initiation site," Journal of Virology, vol. 67, no. 1, pp. 415-424, 1993.

[32] A. K. Boehm, A. Saunders, J. Werner, and J. T. Lis, "Transcription factor and polymerase recruitment, modification, and movement on dhsp70 in vivo in the minutes following heat shock," Molecular and Cellular Biology, vol. 23, no. 21, pp. 7628-7637, 2003.

[33] C. Cheng and P. A. Sharp, "RNA polymerase II accumulation in the promoter-proximal region of the dihydrofolate reductase and $\gamma$-actin genes," Molecular and Cellular Biology, vol. 23, no. 6, pp. 1961-1967, 2003. 
[34] H. P. Phatnani and A. L. Greenleaf, "Phosphorylation and functions of the RNA polymerase II CTD," Genes and Development, vol. 20, no. 21, pp. 2922-2936, 2006.

[35] M. Becker, C. Baumann, S. John et al., "Dynamic behavior of transcription factors on a natural promoter in living cells," EMBO Reports, vol. 3, no. 12, pp. 1188-1194, 2002.

[36] M. Sawadogo and R. G. Roeder, "Factors involved in specific transcription by human RNA polymerase II: analysis by a rapid and quantitative in vitro assay," Proceedings of the National Academy of Sciences of the United States of America, vol. 82, no. 13, pp. 4394-4398, 1985.

[37] D. K. Hawley and R. G. Roeder, "Functional steps in transcription initiation and reinitiation from the major late promoter in a HeLa nuclear extract," Journal of Biological Chemistry, vol. 262, no. 8, pp. 3452-3461, 1987.

[38] M. N. Szentirmay and M. Sawadogo, "Sarkosyl block of transcription reinitiation by RNA polymerase II as visualized by the colliding polymerases reinitiation assay," Nucleic Acids Research, vol. 22, no. 24, pp. 5341-5346, 1994.

[39] S. M. Dehm, T. L. Hilton, E. H. Wang, and K. Bonham, "SRC proximal and core promoter elements dictate TAF1 dependence and transcriptional repression by histone deacetylase inhibitors," Molecular and Cellular Biology, vol. 24, no. 6, pp. 2296-2307, 2004

[40] C. L. Kostyniuk, S. M. Dehm, D. Batten, and K. Bonham, "The ubiquitous and tissue specific promoters of the human SRC gene are repressed by inhibitors of histone deacetylases," Oncogene, vol. 21, no. 41, pp. 6340-6347, 2002.

[41] J. E. F. Butler and J. T. Kadonaga, "Enhancer-promoter specificity mediated by DPE or TATA core promoter motifs," Genes and Development, vol. 15, no. 19, pp. 2515-2519, 2001.

[42] T. Juven-Gershon, J. Y. Hsu, and J. T. Kadonaga, "Caudal, a key developmental regulator, is a DPE-specific transcriptional factor," Genes and Development, vol. 22, no. 20, pp. 2823-2830, 2008.

[43] M. C. Thomas and C. M. Chiang, "The general transcription machinery and general cofactors," Critical Reviews in Biochemistry and Molecular Biology, vol. 41, no. 3, pp. 105-178, 2006.

[44] S. Malik and R. G. Roeder, "Dynamic regulation of pol II transcription by the mammalian Mediator complex," Trends in Biochemical Sciences, vol. 30, no. 5, pp. 256-263, 2005.

[45] X. Jacq, C. Brou, Y. Lutz, I. Davidson, P. Chambon, and L. Tora, "Human TAF(II)30 is present in a distinct TFIID complex and is required for transcriptional activation by the estrogen receptor," Cell, vol. 79, no. 1, pp. 107-117, 1994.

[46] W. Chen, I. Rogatsky, and M. J. Garabedian, "MED14 and MED1 differentially regulate target-specific gene activation by the glucocorticoid receptor," Molecular Endocrinology, vol. 20, no. 3, pp. 560-572, 2006.

[47] W. Chen and R. G. Roeder, "The Mediator subunit MED1/TRAP220 is required for optimal glucocorticoid receptor-mediated transcription activation," Nucleic Acids Research, vol. 35, no. 18, pp. 6161-6169, 2007.

[48] N. Yudkovsky, J. A. Ranish, and S. Hahn, "A transcription reinitiation intermediate that is stabilized by activator," Nature, vol. 408, no. 6809, pp. 225-229, 2000.

[49] R. Sandaltzopoulos and P. B. Becker, "Heat shock factor increases the reinitiation rate from potentiated chromatin templates," Molecular and Cellular Biology, vol. 18, no. 1, pp. 361-367, 1998.

[50] W. L. Kraus and J. T. Kadonaga, "p300 and estrogen receptor cooperatively activate transcription via differential enhancement of initiation and reinitiation," Genes and Development, vol. 12 , no. 3, pp. 331-342, 1998.
[51] P. L. Sheridan, T. P. Mayall, E. Verdin, and K. A. Jones, "Histone acetyltransferases regulate HIV-1 enhancer activity in vitro," Genes and Development, vol. 11, no. 24, pp. 33273340, 1997.

[52] P. A. Wade, D. Pruss, and A. P. Wolffe, "Histone acetylation: chromatin in action," Trends in Biochemical Sciences, vol. 22, no. 4, pp. 128-132, 1997.

[53] M. T. Knuesel, K. D. Meyer, C. Bernecky, and D. J. Taatjes, "The human CDK8 subcomplex is a molecular switch that controls Mediator coactivator function," Genes and Development, vol. 23, no. 4, pp. 439-451, 2009.

[54] D. J. Taatjes, "The human Mediator complex: a versatile, genome-wide regulator of transcription," Trends in Biochemical Sciences, vol. 35, no. 6, pp. 315-322, 2010. 

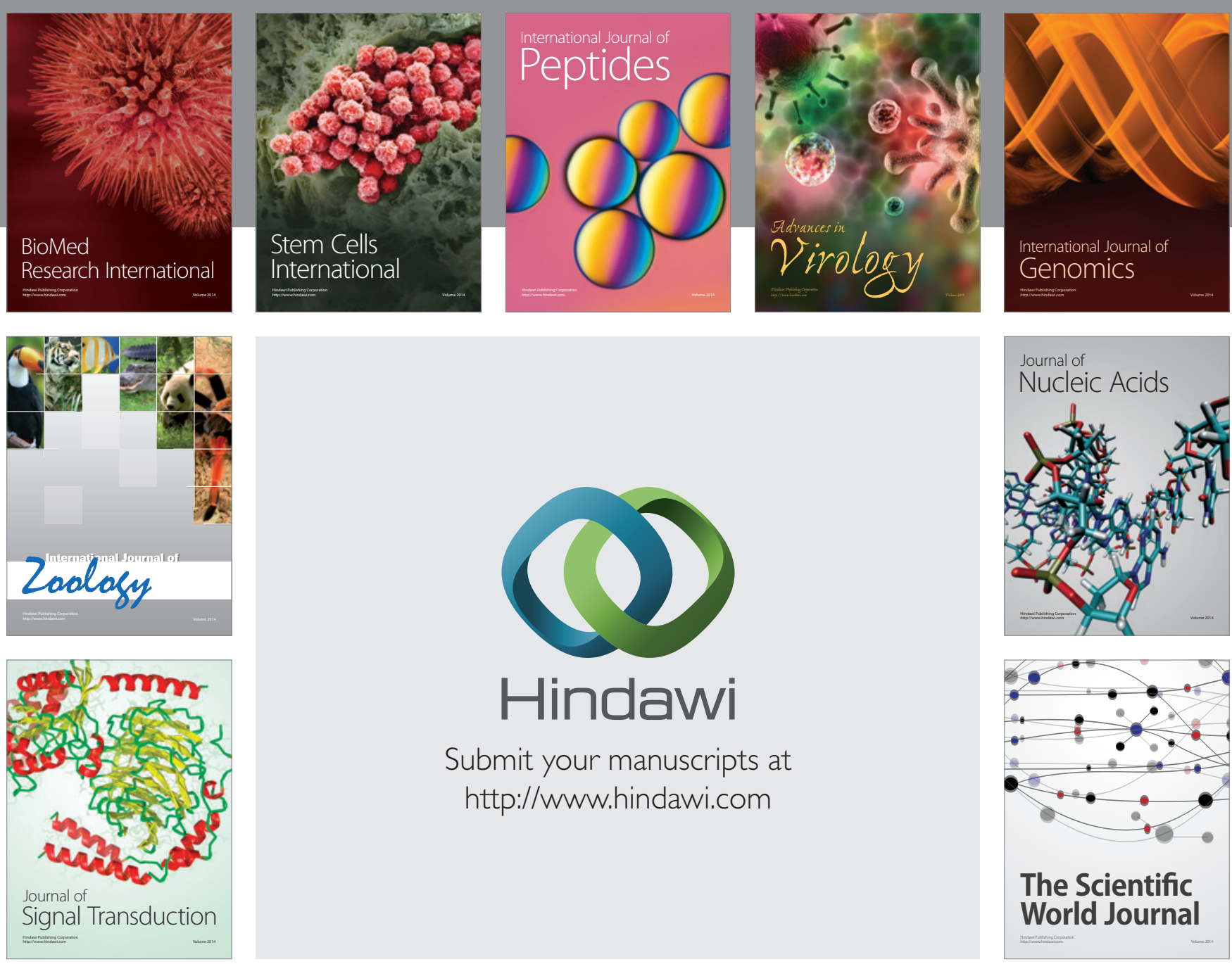

Submit your manuscripts at

http://www.hindawi.com
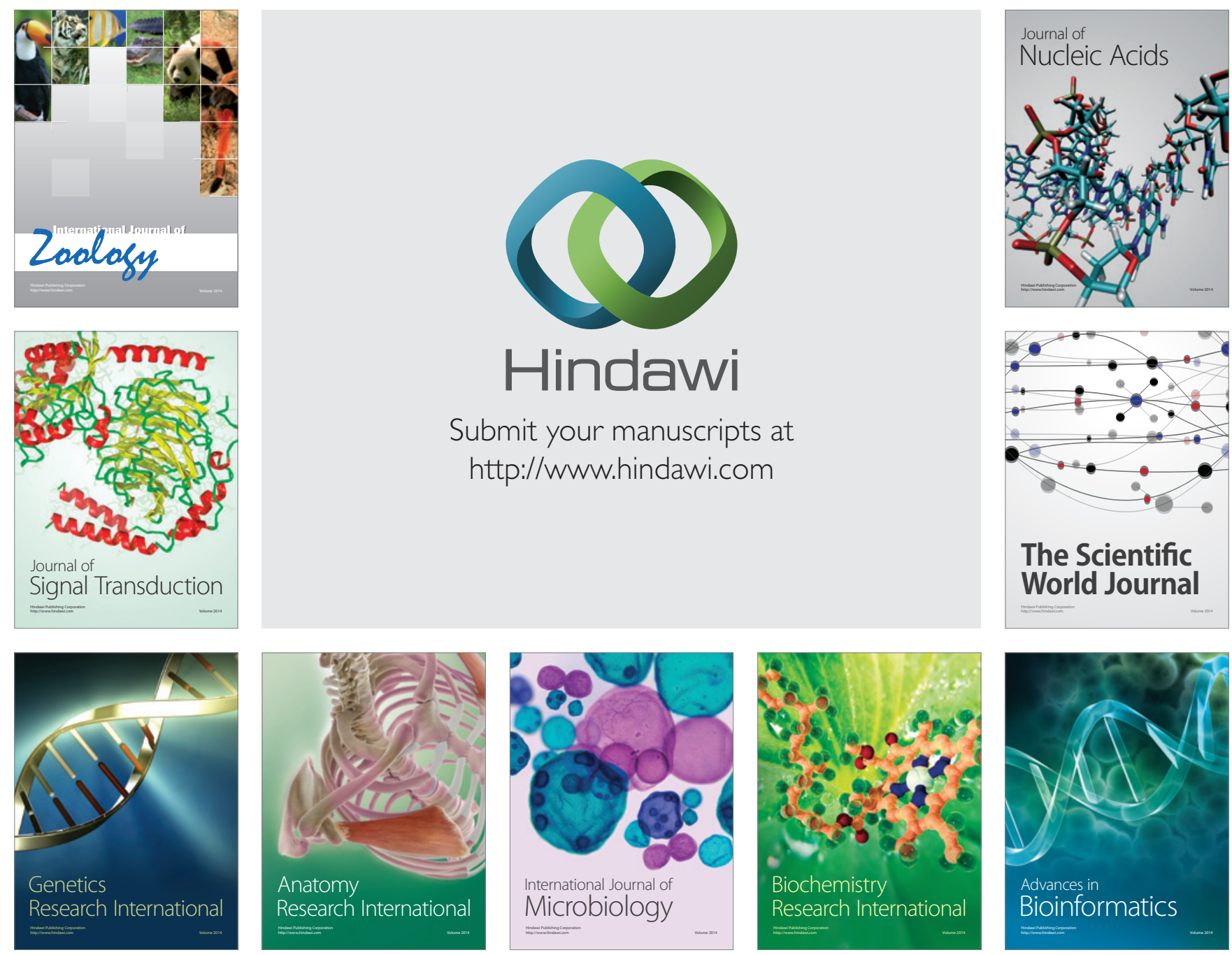

The Scientific World Journal
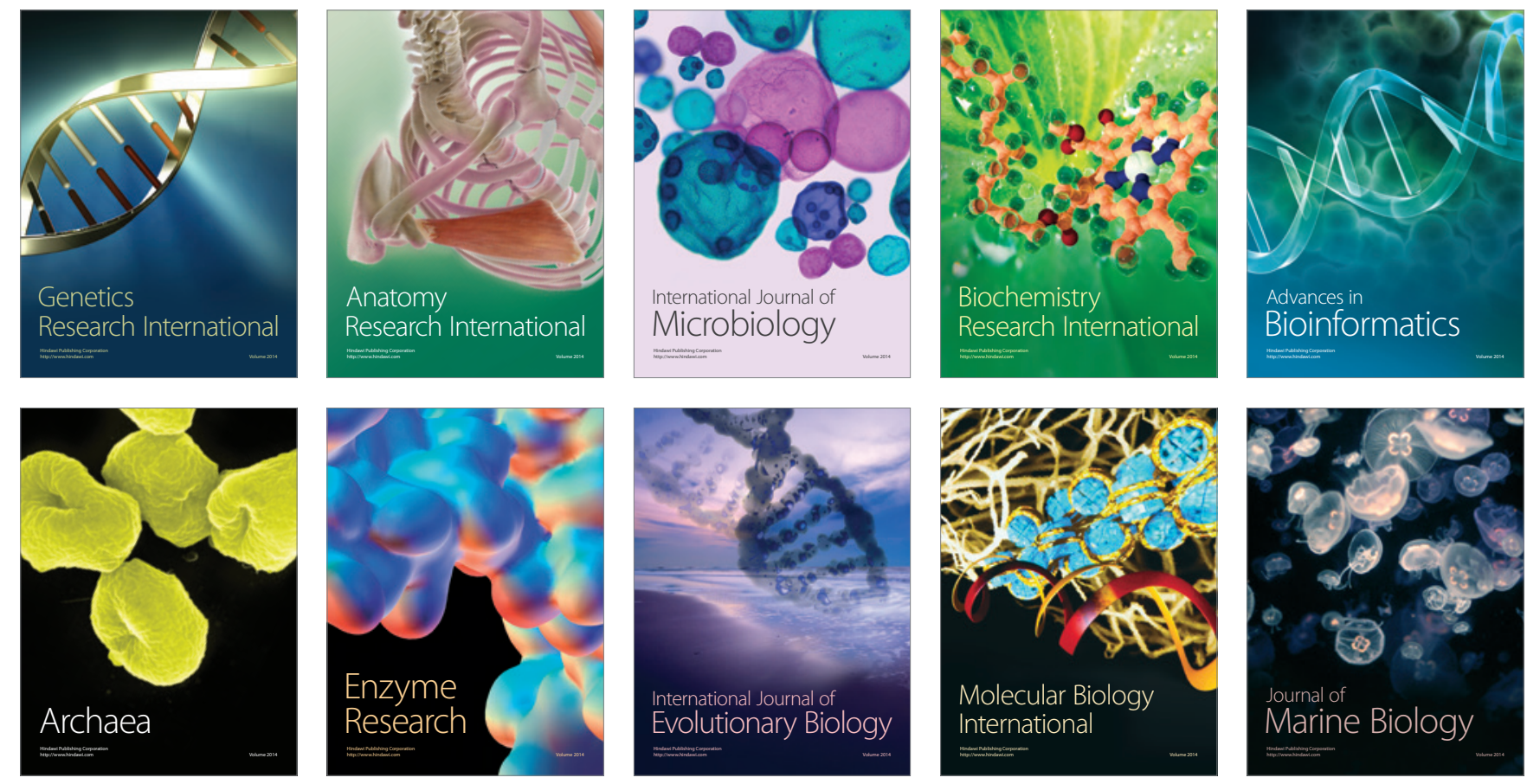